\title{
Mixture of Inverse Weibull and Lognormal Distributions: Properties, Estimation, and Illustration
}

\author{
K. S. Sultan ${ }^{1}$ and A. S. Al-Moisheer ${ }^{2}$ \\ ${ }^{1}$ Department of Statistics and Operations Research, College of Science, King Saud University, P.O. Box 2455, Riyadh 11451, Saudi Arabia \\ ${ }^{2}$ Department of Mathematics, College of Science, Al Jouf University, P.O. Box 848, Sakaka 42421, Saudi Arabia
}

Correspondence should be addressed to K. S. Sultan; ksultan@ksu.edu.sa

Received 26 December 2014; Accepted 21 March 2015

Academic Editor: Gen Qi Xu

Copyright @ 2015 K. S. Sultan and A. S. Al-Moisheer. This is an open access article distributed under the Creative Commons Attribution License, which permits unrestricted use, distribution, and reproduction in any medium, provided the original work is properly cited.

We discuss the two-component mixture of the inverse Weibull and lognormal distributions (MIWLND) as a lifetime model. First, we discuss the properties of the proposed model including the reliability and hazard functions. Next, we discuss the estimation of model parameters by using the maximum likelihood method (MLEs). We also derive expressions for the elements of the Fisher information matrix. Next, we demonstrate the usefulness of the proposed model by fitting it to a real data set. Finally, we draw some concluding remarks.

\section{Introduction}

Finite mixture models have continued to receive increasing attention over the years from both practical and theoretical points of view. As stated by Al-Hussaini and Sultan [1], direct applications of the finite mixture models are in many fields of science and engineering. Indirect applications of the mixture models include outliers, cluster analysis, latent structure models, modeling of prior densities, empirical Bayes method, and nonparametric (kernel) density estimation. For detailed discussions on properties, estimation methods, and applications of finite mixture distributions, one may refer to Everitt and Hand [2], Titterington et al. [3], McLachlan and Basford [4], Lindsay [5], McLachlan and Krishnan [6], and McLachlan and Peel [7]. Al-Hussaini and Sultan [1] have reviewed the properties and estimation techniques for finite mixtures of some lifetime models. In this paper, we study the two-component mixture of inverse Weibull and lognormal distributions as a lifetime model and discuss its reliability properties as well as the MLE estimation method.

The MIWLND has its pdf as

$$
\begin{gathered}
f(t ; \Theta)=p_{1} f_{1}\left(t ; \Theta_{1}\right)+p_{2} f_{2}\left(t ; \Theta_{2}\right), \\
0<p_{1}, \quad p_{2}<1, \quad p_{1}+p_{2}=1,
\end{gathered}
$$

where the pdf of the first component (inverse Weibull) is given by

$$
f_{1}\left(t ; \Theta_{1}\right)=\beta \alpha^{-\beta} t^{-(\beta+1)} e^{-(\alpha t)^{-\beta}}, \quad t \geq 0, \alpha, \beta>0,
$$

and the pdf of the second component (lognormal) is given by

$$
\begin{array}{r}
f_{2}\left(t ; \Theta_{2}\right)=\frac{1}{\sqrt{2 \pi} \sigma t} e^{-(1 / 2)((\log t-\mu) / \sigma)^{2}}, \\
t \geq 0, \quad-\infty<\mu<\infty, \quad \sigma>0,
\end{array}
$$

$\Theta=\left(p_{1}, \alpha, \beta, \mu, \sigma\right), \Theta_{1}=(\alpha, \beta)$, and $\Theta_{2}=(\mu, \sigma)$. For an excellent discussion on the properties of these two mixing distributions and related inferential procedures, one may refer to Johnson et al. [8].

Evidently, the cdf of the MIWLND is given by

$$
F(t ; \Theta)=p_{1} F_{1}\left(t ; \Theta_{1}\right)+p_{2} F_{2}\left(t ; \Theta_{2}\right),
$$

where

$$
\begin{gathered}
F_{1}\left(t ; \Theta_{1}\right)=e^{-(\alpha t)^{-\beta}}, \quad t \geq 0, \alpha, \beta>0, \\
F_{2}\left(t ; \Theta_{2}\right)=\Phi\left(\frac{\log t-\mu}{\sigma}\right), \quad t \geq 0,-\infty<\mu<\infty, \sigma>0,
\end{gathered}
$$

with $\Phi(\cdot)$ being the cdf of the standard normal distribution. 
As pointed out by Crow and Shimizu [9] and Johnson et al. [8], the lognormal distribution has found important applications in a wide variety of fields. Some recent articles dealing with lognormal distribution include the works of Kim and Yum [10] and Lin et al. [11]. Mixture models of two lognormal distributions have been discussed by Al-Hussaini et al. [12]. The inverse Weibull distribution has been fitted for some pieces of data from reliability engineering and biomedical studies; see Drapella [13]. Recently, Sultan et al. [14, 15] have discussed some properties of a mixture of two inverse Weibull distributions and the hypotheses testing problem regarding the number of components.

It may be noted that, while the lognormal and inverse Weibull distributions are always unimodal, mixing an inverse Weibull distribution with a lognormal distribution produces a model with a flexible hazard function which covers both unimodal and bimodal shapes and therefore has a great potential while fitting practical data.

The rest of this paper is organized as follows. In Section 2, we discuss some basic properties of the MIWLND. In Section 3, we discuss the problem of estimating all five unknown parameters of the MIWLND in (1) through the method of maximum likelihood. In Section 4, we illustrate the usefulness of the proposed model by fitting it to a real dataset. We derive expressions for the elements of the Fisher information matrix, and these are presented in the appendix. Finally, we draw some concluding remarks in Section 5.

\section{Some Properties}

Keller et al. [16] and Jiang et al. [17] have discussed some properties of the inverse Weibull distribution in (2), while properties of the lognormal distribution in (3) are rather wellknown; see, for example, Crow and Shimizu [9] and Johnson et al. [8]. In this section, we discuss some properties of the MIWLND by combining the corresponding results of the inverse Weibull and lognormal distributions.

2.1. Mean and Variance. The mean of the MIWLND in (1) is simply given by

$$
E(T)=\frac{p_{1}}{\alpha} \Gamma\left(1-\frac{1}{\beta}\right)+p_{2} e^{\mu+\sigma^{2} / 2}, \quad \text { for } \beta>1,
$$

while the variance is given by

$$
\begin{aligned}
\operatorname{Var}(T)= & \frac{p_{1}}{\alpha^{2}}\left[\Gamma\left(1-\frac{2}{\beta}\right)-p_{1} \Gamma^{2}\left(1-\frac{1}{\beta}\right)\right] \\
& +p_{2} e^{2 \mu+\sigma^{2}}\left[e^{\sigma^{2}}-p_{2}\right] \\
& -\frac{2 p_{1} p_{2}}{\alpha} \Gamma\left(1-\frac{1}{\beta}\right) e^{\mu+\sigma^{2} / 2}, \quad \text { for } \beta>2,
\end{aligned}
$$

where $\Gamma(\cdot)$ denotes the complete gamma function.
TABLE 1: The mode(s) and median of the MIWLND.

\begin{tabular}{lcc}
\hline$\Theta=\left(p_{1}, \alpha, \beta, \mu, \sigma\right)$ & Mode $(\mathrm{s})$ & Median \\
\hline $0.2,2,1,2,1$ & 0.2561 & 5.6778 \\
$0.3,2,1,2,1$ & 0.2535 & 4.7407 \\
$0.5,2,1,2,1$ & 0.2514 & 2.7830 \\
$0.6,2,1,2,1$ & 0.2511 & 1.9410 \\
$0.8,2,1,2,1$ & 0.2503 & 1.0415 \\
\hline $0.2,1.5,1,1,0.12$ & $0.3333,2.6785$ & 25.1648 \\
$0.3,1.5,1,1,0.12$ & $0.3333,2.6778$ & 9.3579 \\
$0.5,1.5,1,1,0.12$ & $0.3333,2.6755$ & 0.6827 \\
$0.6,1.5,1,1,0.12$ & $0.3333,2.6735$ & 0.5563 \\
$0.8,1.5,1,1,0.12$ & $0.3333,2.6633$ & 0.4735 \\
\hline
\end{tabular}

2.2. Mode and Median. The mode (modes) of the MIWLND is (are) obtained by solving the following nonlinear equation with respect to $t$ :

$$
\begin{aligned}
& p_{1} \beta \alpha^{-\beta} t^{-(\beta+2)} e^{-(\alpha t)^{-\beta}}\left[-(\beta+1)+\beta \alpha^{-\beta} t^{-(\beta)}\right] \\
& -p_{2} \frac{1}{\sqrt{2 \pi} \sigma^{3} t^{2}} e^{-(1 / 2)((\log t-\mu) / \sigma)^{2}}\left[\sigma^{2}+(\log t-\mu)\right]=0 .
\end{aligned}
$$

From (4), the median of the MIWLND is obtained by solving the following nonlinear equation with respect to $t$ :

$$
p_{1} e^{-\left(\alpha_{1} t\right)^{-\beta_{1}}}+p_{2} \Phi\left(\frac{\log t-\mu}{\sigma}\right)=0.5 \text {. }
$$

Table 1 presents the modes and median of the MIWLND for some selected choices of the parameters.

The values of the parameters $p_{1}, \alpha, \beta, \mu$, and $\sigma$ in Table 1 were chosen suitably so as to demonstrate the unimodal and bimodal cases of the density function of the proposed mixture model. From Table 1, we see that the modes are only slightly affected by changes in the value of the mixing proportion $p_{1}$, while the median changes significantly with $p_{1}$. Figures $1(a)$ and 2(a) display some typical shapes of the pdf of the MIWLND.

2.3. Reliability and Failure Rate Functions. The reliability function (survival function) of the MIWLND is evidently

$$
R(t)=p_{1}\left[1-e^{-(\alpha t)^{-\beta}}\right]+p_{2}\left[1-\Phi\left(\frac{\log t-\mu}{\sigma}\right)\right], \quad t \geq 0 .
$$

By using (3) and (4), the failure rate function (hazard rate function HRF) of the MIWLND is given by

$$
\begin{aligned}
& r(t)=\left(p_{1} \beta \alpha^{-\beta} t^{-(\beta+1)} e^{-(\alpha t)^{-\beta}}\right. \\
&\left.+p_{2} \frac{1}{\sqrt{2 \pi} \sigma t} e^{-(1 / 2)((\log t-\mu) / \sigma)^{2}}\right) \\
& \cdot\left(p_{1}\left(1-e^{-(\alpha t)^{-\beta}}\right)+p_{2}\left(1-\Phi\left(\frac{\log t-\mu}{\sigma}\right)\right)\right)^{-1}, \\
& t \geq 0,
\end{aligned}
$$




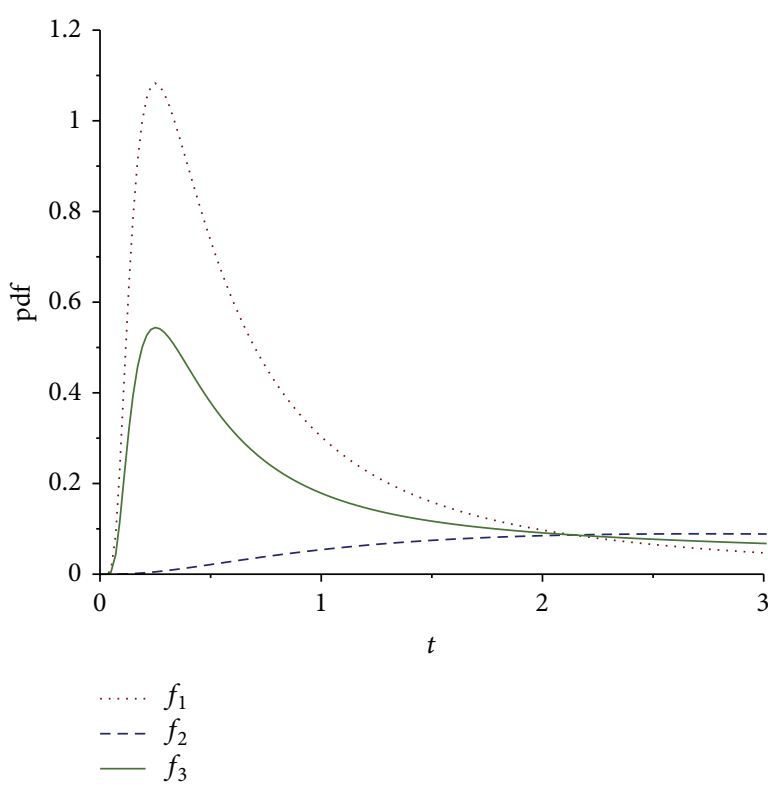

(a)
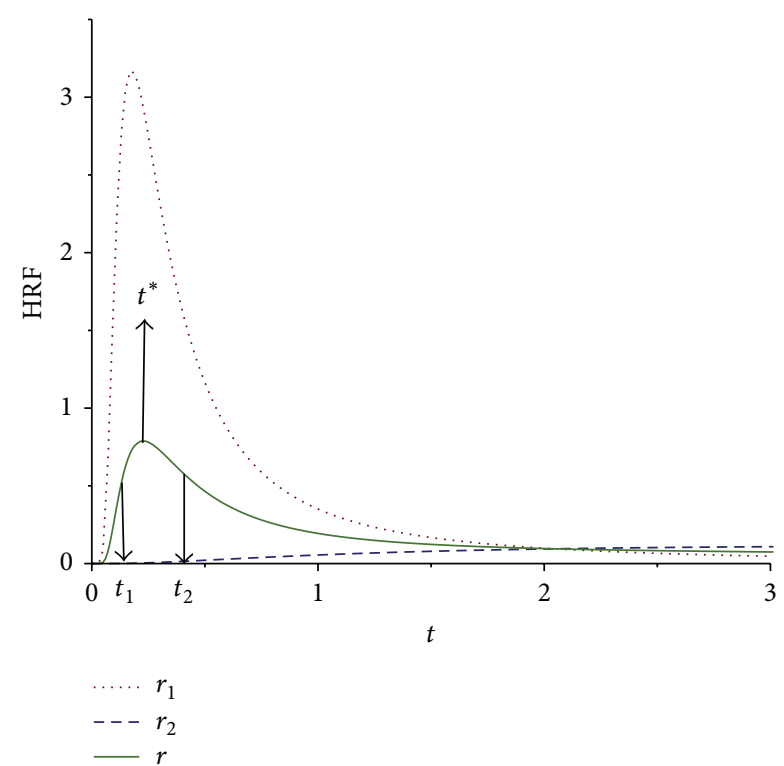

FiguRE 1: (a) Density functions components and their mixture with parameters $(0.5,2,1,2,1)$-unimodal case. (b) HR functions: components and their mixture with parameters $(0.5,2,1,2,1)$ - unimodal case.

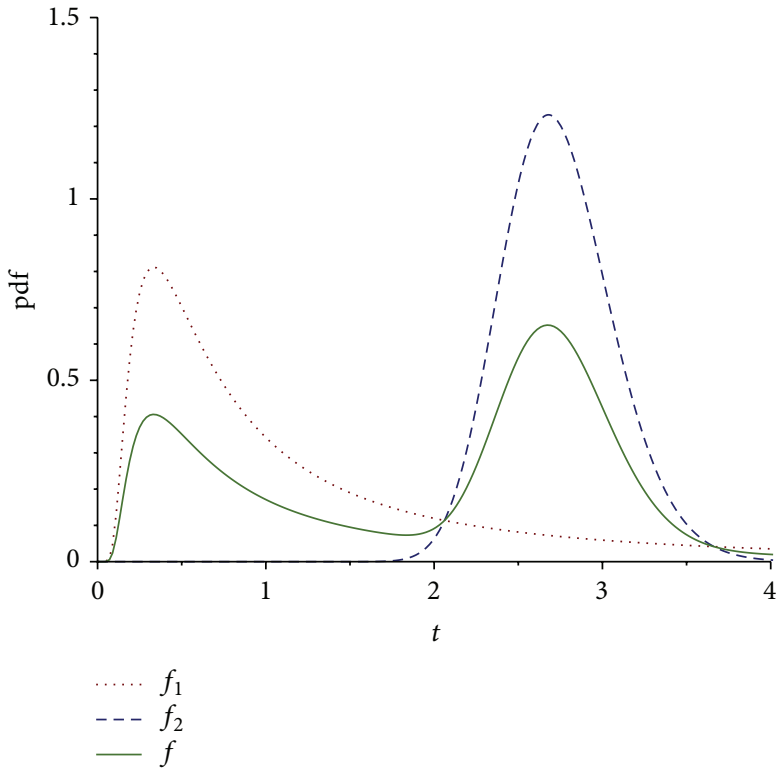

(a)

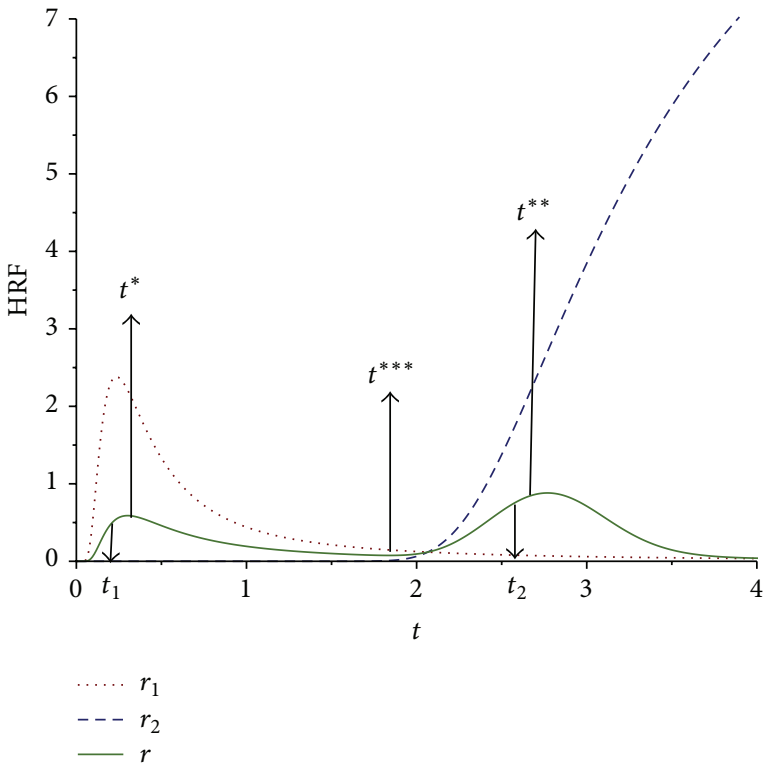

(b)

FIgURE 2: (a) Density functions: components and their mixture with parameters $(0.5,1.5,1,1,0.12)$ - bimodal case. (b) HR functions: components and their mixture with parameters $(0.5,1.5,1,1,0.12)$-bimodal case.

which can be expressed, in view of the result by Al-Hussaini and Sultan [1], as

$$
r(t)=h(t) r_{1}(t)+(1-h(t)) r_{2}(t),
$$

where

$$
h(t)=\frac{1}{1+p_{2} R_{2}(t) / p_{1} R_{1}(t)}, \quad r_{i}(t)=\frac{f_{i}(t)}{R_{i}(t)}, \quad i=1,2,
$$

$$
R_{1}(t)=1-e^{-(\alpha t)^{-\beta}}, \quad R_{2}(t)=1-\Phi\left(\frac{\log t-\mu}{\sigma}\right) .
$$

The derivative of the hazard rate function is then given by

$$
\begin{aligned}
r^{\prime}(t)= & h(t) r_{1}^{\prime}(t)+(1-h(t)) r_{2}^{\prime}(t) \\
& -h(t)(1-h(t))\left[r_{1}(t)-r_{2}(t)\right]^{2} .
\end{aligned}
$$


The failure rate function of the MIWLND in (11) possesses the following limits.

Lemma 1. One has

$$
\begin{aligned}
& \lim _{t \rightarrow 0} r(t)=0, \\
& \lim _{t \rightarrow \infty} r(t)=0 .
\end{aligned}
$$

Proof. It can be shown that $\lim _{t \rightarrow 0} r_{i}(t)=0$ and $\lim _{t \rightarrow \infty} r_{i}(t)=0$, for $i=1,2$; see Sultan et al. [14]. Then, from (13), it can be shown that

$$
\lim _{t \rightarrow 0} h(t)=p_{1}
$$

and thus (15) is proved.

Once again, from (13), we note that $p_{2} R_{2}(t) / p_{1} R_{1}(t) \geq$ 0 and that $\lim _{t \rightarrow \infty}\left(p_{2} R_{2}(t) / p_{1} R_{1}(t)\right) \neq-1$. It follows that $|h(t)|<\infty$ and so (16) is proved.

2.4. Properties of the Failure Rate Function. Suppose $t_{1}=$ $\min \left(t_{1}^{*}, t_{2}^{*}\right)$ and $t_{2}=\max \left(t_{1}^{*}, t_{2}^{*}\right)$, where, for $i=1,2, t_{i}^{*}$ represents the mode of the density function $f_{i}(t)$. From $r_{i}(t)=$ $f_{i}(t) / R_{i}(t)$, we see that both $f_{1}(t)$ and $f_{2}(t)$ in the numerator of $r_{i}(t)$ increase in $\left(0, t_{1}\right)$, whereas the denominator decreases in the same interval. So $r(t)$ increases in $\left(0, t_{1}\right)$. Moreover, as $t \rightarrow \infty, r(t) \rightarrow 0$. For this reason, in the interval $\left(t_{1}, \infty\right)$ two cases arise.

(a) Unimodal Case. Suppose $t^{*}$ is the maximum point of the failure rate of the mixture. When the difference $\Delta$ between $r_{1}(t)$ and $r_{2}(t)$ in the interval $\left(t_{1}, t^{*}\right)$ is small so that the first two terms of $r^{\prime}(t)$ in (14) dominate the third term, then $r^{\prime}(t)>$ 0 in $\left(t_{1}, t^{*}\right)$. When the difference $\Delta$ increases to the point that the third term in $r^{\prime}(t)$ dominates the first two terms, then $r^{\prime}(t)<0$ in $\left(t^{*}, \infty\right)$. Summarizing, we have the failure rate of the MIWLND increasing in $\left(0, t^{*}\right)$ and decreasing in $\left(t^{*}, \infty\right)$, reaching zero as $t \rightarrow \infty$; see Figure 1(b), for example.

(b) Bimodal Case. Suppose $t^{*}$ and $t^{* *}$ denote, respectively, the smallest and the largest maximum points of the failure rate of the mixture. When the difference $\Delta$ between $r_{1}(t)$ and $r_{2}(t)$ in the interval $\left(t_{1}, t^{*}\right)$ is small, where $t_{1}<t^{*}<t_{2}<t^{* *}$, the third term in (14) is dominated by the first two terms and so $r^{\prime}(t)>0$ in $\left(0, t^{*}\right)$. The difference $\Delta$ in the interval $\left(t^{*}, t^{* * *}\right)$, where $t^{* * *}$ is the local minimum point of $r(t)$, becomes larger to the point that the third term in $r^{\prime}(t)$ dominates the first two terms resulting in $r^{\prime}(t)<0$ in $\left(t^{*}, t^{* * *}\right)$. In the interval $\left(t^{* * *}, t^{* *}\right)$, the difference becomes small so that the third term in $r^{\prime}(t)$ is dominated by the first two terms and so $r^{\prime}(t)>0$. Summarizing, we have the failure rate of the mixed model increasing in $\left(0, t^{*}\right)$, decreasing in $\left(t^{*}, t^{* * *}\right)$, increasing in $\left(t^{* * *}, t^{* *}\right)$, and decreasing again in $\left(t^{* *}, \infty\right)$, reaching 0 as $t$ tends to $\infty$; see Figure 2(b), for example.

As we can see from Figures 1(a), 1(b), 2(a), and 2(b), the shape of the model (unimodal and bimodal) is affected by the parameters choices. For example, when $(\alpha, \mu, \sigma)$ changes from $(2.0,2.0,1.0)$ to $(1.5,1.5,0.12)$ the model is changed from the unimodal case to the bimodal case.

\section{Maximum Likelihood Estimation}

In this section, we describe the ML approach for the estimation of the 5-dimensional parameter vector $\Theta$ of the mixture density in (1) based on a random sample of size $n$. The MLE $\widehat{\Theta}$ is obtained as the solution of the likelihood equations:

$$
\frac{\partial L(\Theta)}{\partial \theta_{i}}=0, \quad i=1,2,3,4,5,
$$

or, equivalently,

$$
\frac{\partial \log L(\Theta)}{\partial \theta_{i}}=0, \quad i=1,2,3,4,5,
$$

where

$$
L(\Theta)=\prod_{j=1}^{n} f\left(t_{j} ; \Theta\right)
$$

is the likelihood function formed under the assumption of iid data $t_{1}, \ldots, t_{n}$. The likelihood function corresponding to the mixture density in (1) is then given by

$$
L(\Theta)=\prod_{j=1}^{n}\left[p_{1} f_{1}\left(t_{j} ; \Theta_{1}\right)+p_{2} f_{2}\left(t_{j} ; \Theta_{2}\right)\right],
$$

where $\Theta_{1}=(\alpha, \beta)$ and $\Theta_{2}=(\mu, \sigma)$.

By differentiating the log-likelihood function $L^{*}=$ $\log L(\Theta)$ with respect to the five parameters of the model, we get the first order derivatives of $L^{*}$ to be

$$
\begin{gathered}
\frac{\partial L^{*}}{\partial \theta_{1}}=\frac{\partial L^{*}}{\partial p_{1}}=\sum_{j=1}^{n} \omega\left(t_{j} ; \Theta\right)=0, \\
\frac{\partial L^{*}}{\partial \theta_{2}}=\frac{\partial L^{*}}{\partial \alpha}=\sum_{j=1}^{n} p_{1} \phi_{1}\left(t_{j} ; \Theta\right) \eta_{1}\left(t_{j} ; \Theta\right)=0, \\
\frac{\partial L^{*}}{\partial \theta_{3}}=\frac{\partial L^{*}}{\partial \beta}=\sum_{j=1}^{n} p_{1} \phi_{2}\left(t_{j} ; \Theta\right) \eta_{1}\left(t_{j} ; \Theta\right)=0, \\
\frac{\partial L^{*}}{\partial \theta_{4}}=\frac{\partial L^{*}}{\partial \mu}=\sum_{j=1}^{n} p_{2} \psi_{1}\left(t_{j} ; \Theta\right) \eta_{2}\left(t_{j} ; \Theta\right)=0, \\
\frac{\partial L^{*}}{\partial \theta_{5}}=\frac{\partial L^{*}}{\partial \sigma}=\sum_{j=1}^{n} p_{2} \psi_{2}\left(t_{j} ; \Theta\right) \eta_{2}\left(t_{j} ; \Theta\right)=0,
\end{gathered}
$$

where $\omega\left(t_{j} ; \Theta\right), \quad \phi_{1}\left(t_{j} ; \Theta\right), \quad \phi_{2}\left(t_{j} ; \Theta\right), \quad \eta_{1}\left(t_{j} ; \Theta\right), \quad \eta_{2}\left(t_{j} ; \Theta\right)$, $\psi_{1}\left(t_{j} ; \Theta\right)$, and $\psi_{2}\left(t_{j} ; \Theta\right)$ are as follows:

$$
\begin{gathered}
\omega\left(t_{j} ; \Theta\right)=\frac{f_{1}\left(t_{j} ; \Theta_{1}\right)-f_{2}\left(t_{j} ; \Theta_{2}\right)}{f\left(t_{j} ; \Theta\right)}, \\
\phi_{1}\left(t_{j} ; \Theta\right)=-\beta \alpha^{-1}+\beta \alpha^{-(\beta+1)}\left(t_{j}\right)^{-\beta},
\end{gathered}
$$


TABLE 2

\begin{tabular}{ccccccccc}
\hline Maintenance data & $N$ & Mean & Mode & Median & Variance & Standard deviation & Minimum & Maximum \\
\hline & 46 & 3.8499 & 0.3182 & 1.4670 & 59.1756 & 7.6926 & 0.2 & 24.5 \\
\hline
\end{tabular}

$$
\begin{gathered}
\phi_{2}\left(t_{j} ; \Theta\right)=\beta^{-1}-\log (\alpha)-\log \left(t_{j}\right)+\left(\alpha t_{j}\right)^{-\beta} \log \left(\alpha t_{j}\right), \\
\eta_{1}\left(t_{j} ; \Theta\right)=\frac{f_{1}\left(t_{j} ; \Theta\right)}{f\left(t_{j} ; \Theta\right)} \\
\eta_{2}\left(t_{j} ; \Theta\right)=\frac{f_{2}\left(t_{j} ; \Theta\right)}{f\left(t_{j} ; \Theta\right)} \\
\psi_{1}\left(t_{j} ; \Theta\right)=\frac{\left(\log t_{j}-\mu\right)}{\sigma^{2}} \\
\psi_{2}\left(t_{j} ; \Theta\right)=\left(\log t_{j}-\mu\right)^{2} \sigma^{-3}-\sigma^{-1}
\end{gathered}
$$

and $f\left(t_{j} ; \Theta\right), f_{1}\left(t_{j} ; \Theta_{1}\right)$, and $f_{2}\left(t_{j} ; \Theta_{2}\right)$ are as in (1), (2), and (3), respectively. The maximum likelihood estimates of the five parameters may be obtained by solving (22) by using a numerical method such as the Newton-Raphson method.

\section{Data Analysis}

In this section, we use a real data set to illustrate the usefulness of the proposed mixture model. The following maintenance data were reported on active repair times (hours) for an airborne communication transceiver (see Von Alven [18, page 156]): $0.2,0.3,0.5,0.5,0.5,0.5,0.6,0.6,0.7,0.7,0.7,0.8,0.8,1.0$, $1.0,1.0,1.0,1.1,1.3,1.5,1.5,1.5,1.5,2.0,2.0,2.2,2.5,2.7,3.0,3.0$, 3.3, 3.3, 4.0, 4.0, 4.5, 4.7, 5.0, 5.4, 5.4, 7.0, 7.5, 8.8, 9.0, 10.3, 22.0, and 24.5.

In Section 2, we introduce some properties of the MIWLND and we use it to analyze this maintenance data. Table 2 is descriptive statistics for maintenance data.

For interpretation of the failure rate function, the maximum point of the failure rate of the mixture is $t^{*}=0.3424$. In addition, $t_{1}=0.3083$ and $t_{2}=0.6123$ represent the mode of the density function of the inverse Weibull and lognormal distributions, respectively. When the difference $\Delta$ between $r_{1}(t)$ and $r_{2}(t)$ in the interval $\left(t_{1}, t^{*}\right)=(0.3083,0.3424)$ is small so that the first two terms of $r^{\prime}(t)$ in (2.35) dominate the third term, then $r^{\prime}(t)>0$ in $(0.3083,0.3424)$. When the difference $\Delta$ increases to the point that the third term in $r^{\prime}(t)$ dominates the first two terms, then $r^{\prime}(t)<0$ in $(0.3424, \infty)$. Summarizing, we have the failure rate of the MIWLND increasing in $(0,0.3424)$ and decreasing in $(0.3424, \infty)$, reaching zero as $t \rightarrow \infty$; see Figure 3 .

In Figure 4, we see the inverse Weibull with its MLEs $(\widehat{\alpha}=$ $0.8851, \widehat{\beta}=1.0127)$ and lognormal normal with its MLEs $(\widehat{\mu}=0.6584, \widehat{\sigma}=1.1018)$ which are separately not good fits for these data. Also, in Figure 4, we have shown the fitted MIWLND model superimposed on the histogram of

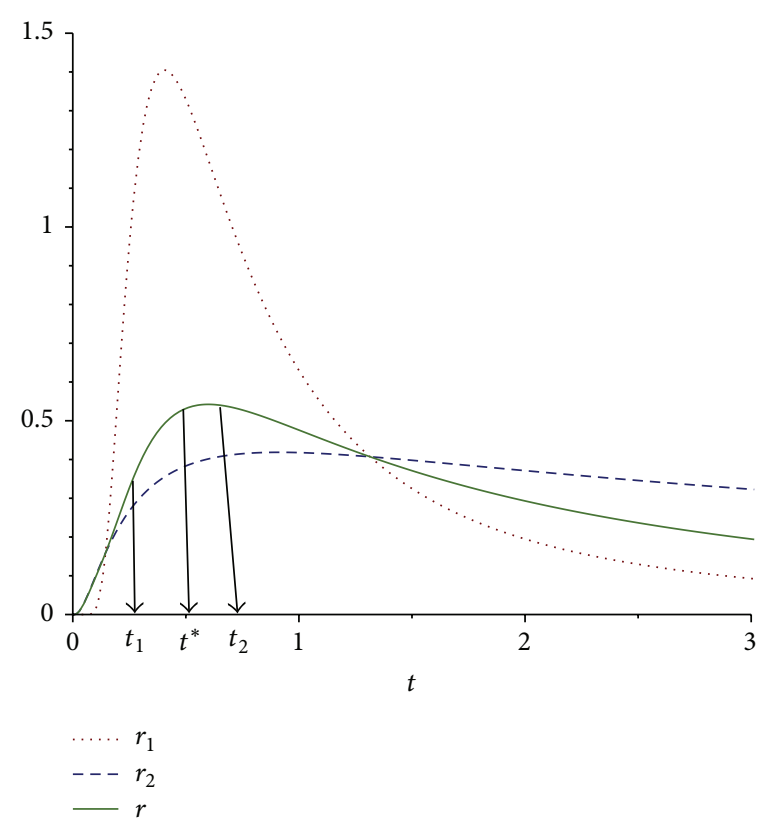

FIGURE 3: Failure rate for the fitted of MIWLN model.

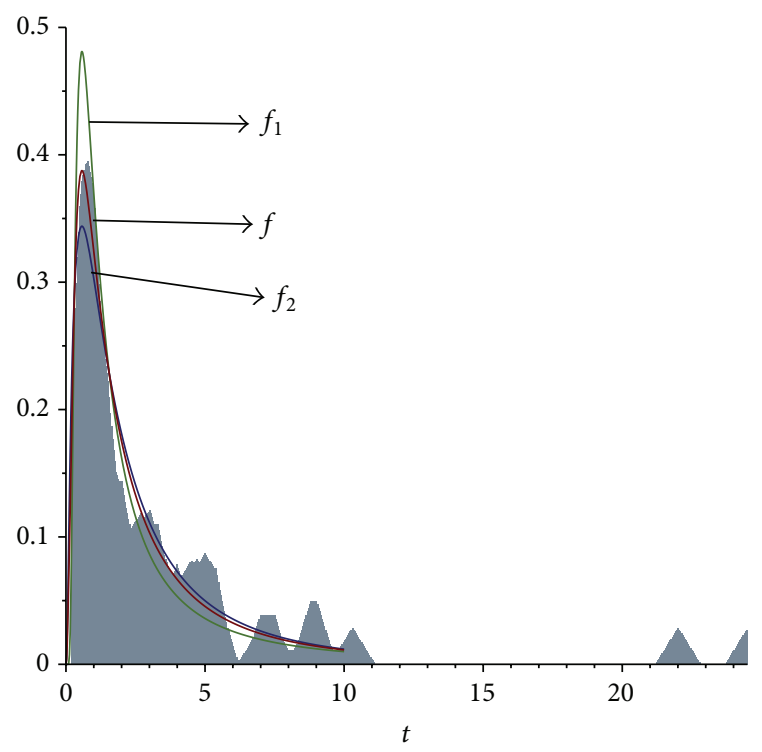

FIgURE 4: Histogram of the data superimposed with the fitted model.

the observed data, which shows that the MIWLND provides a very good fit for these data compared to the individual components.

Further, we use Kolmogorov-Smirnov test (K-S) to fit the data as shown in Table 3. 
TABLE 3

\begin{tabular}{lccc}
\hline Model & Inverse Weibull & Lognormal & MTIWD \\
\hline K-S statistic & 0.5086 & 1.9707 & 0.1688 \\
\hline
\end{tabular}

TABLE 4

\begin{tabular}{lccccc}
\hline Parameter & $\widehat{p}_{1}$ & $\widehat{\alpha}$ & $\widehat{\beta}$ & $\widehat{\mu}$ & $\widehat{\sigma}$ \\
\hline MLE & 0.3181 & 2.4246 & 1.6378 & 0.9365 & 1.1946 \\
\hline Standard error & 0.1208 & 0.6492 & 0.5524 & 0.2161 & 0.1876 \\
\hline
\end{tabular}

It is observed that the K-S distance between the data and the fitted of the MTIWD is 0.1688 which gives a good fit at $1 \%$ level of significance than the inverse Weibull with K-S statistic as 0.5068 and the lognormal with K-S statistic as 1.9709.

Now, the maximum likelihood estimates of the MIWLND parameters with their standard errors were determined as shown in Table 4.

The standard errors of these estimates were calculated by inverting the Fisher information matrix derived in the appendix. The Fisher information $I(\Theta)$ can also be utilized to obtain the approximate $100(1-\delta) \%$ confidence intervals (CIs) of the components of the vectors $\Theta$ as $\widehat{\Theta} \pm \xi_{\delta / 2} \sqrt{V(\widehat{\Theta})}$, where $V(\widehat{\Theta})$ are the variances of the parameters obtained from $I^{-1}(\widehat{\Theta})$, and $\xi_{\delta / 2}$ is the upper $(\delta / 2)$ percentile of the standard normal distribution. The variance-covariance matrix of $\Theta$ was computed as

$$
\begin{aligned}
& I^{-1}(\widehat{\Theta}) \\
& =\left(\begin{array}{ccccc}
0.0146 & -0.0151 & 0.0126 & 0.0089 & -0.0026 \\
-0.0151 & 0.4214 & 0.1063 & -0.0136 & -0.0041 \\
0.0126 & 0.1063 & 0.3052 & 0.0162 & -0.0079 \\
0.0089 & -0.0136 & 0.0162 & 0.0467 & -0.0009 \\
-0.0026 & -0.0041 & -0.0079 & -0.0009 & 0.0352
\end{array}\right) .
\end{aligned}
$$

The $90 \%$ CIs of the parameters that were calculated in this manner are as shown in Table 5.

\section{Concluding Remarks}

In this paper, the MIWLND has been introduced as a lifetime model. Then, the modes and the median of the MIWLND are examined for different choices of the parameters. Also, the behavior of the failure rate function is discussed analytically as well as through some graphs. The estimation of the model parameters by the method of maximum likelihood is then discussed. The estimation method described here is for complete samples. Since most life-testing experiments result in Type I and Type II censored data, it will be of interest to develop inferential methods based on such censored samples. Work in this direction is currently under progress and we hope to report these findings in a future paper.

\section{Appendix}

\section{Fisher Information Matrix}

The likelihood function of $\Theta$ based on the MIWLND is given by

$$
L(\Theta)=\prod_{j=1}^{n}\left[p_{1} f_{1}\left(t_{j} ; \Theta_{1}\right)+p_{2} f_{2}\left(t_{j} ; \Theta_{2}\right)\right],
$$

where $\Theta_{1}=(\alpha, \beta)$ and $\Theta_{2}=(\mu, \sigma)$. By differentiating the $\log$-likelihood function $L^{*}$ with respect to the parameters, we obtain the first order derivatives of $L^{*}$ as given in (22). Upon differentiating these expressions once again with respect to the parameters, we obtain the partial derivatives of second order as follows:

$$
\begin{aligned}
& \frac{\partial^{2} L^{*}}{\partial p_{1}^{2}}=-\sum_{j=1}^{n} \omega^{2}\left(t_{j} ; \Theta\right), \\
& \frac{\partial^{2} L^{*}}{\partial p_{1} \partial \alpha}=\sum_{j=1}^{n} \phi_{1}\left(t_{j} ; \Theta\right) \xi\left(t_{j} ; \Theta\right) \text {, } \\
& \frac{\partial^{2} L^{*}}{\partial p_{1} \partial \beta}=\sum_{j=1}^{n} \phi_{2}\left(t_{j} ; \Theta\right) \xi\left(t_{j} ; \Theta\right) \text {, } \\
& \frac{\partial^{2} L^{*}}{\partial p_{1} \partial \mu}=-\sum_{j=1}^{n} \psi_{1}\left(t_{j} ; \Theta\right) \xi\left(t_{j} ; \Theta\right), \\
& \frac{\partial^{2} L^{*}}{\partial p_{1} \partial \sigma}=-\sum_{j=1}^{n} \psi_{2}\left(t_{j} ; \Theta\right) \xi\left(t_{j} ; \Theta\right) \text {, } \\
& \frac{\partial^{2} L^{*}}{\partial \alpha^{2}}=\sum_{j=1}^{n} p_{1} \eta_{1}\left(t_{j} ; \Theta\right) \\
& \cdot\left[V_{1}\left(t_{j} ; \Theta\right)+\left[1-p_{1} \eta_{1}\left(t_{j} ; \Theta\right)\right] \phi_{1}^{2}\left(t_{j} ; \Theta\right)\right], \\
& \frac{\partial^{2} L^{*}}{\partial \alpha \partial \beta} \\
& =\sum_{j=1}^{n} p_{1} \eta_{1}\left(t_{j} ; \Theta\right)\left[V_{2}\left(t_{j} ; \Theta\right)+\left[1-p_{1} \eta_{1}\left(t_{j} ; \Theta\right)\right]\right. \\
& \left.\cdot \phi_{1}\left(t_{j} ; \Theta\right) \phi_{2}\left(t_{j} ; \Theta\right)\right] \\
& \frac{\partial^{2} L^{*}}{\partial \alpha \partial \mu}=-\sum_{j=1}^{n} p_{1} p_{2} \phi_{1}\left(t_{j} ; \Theta\right) \psi_{1}\left(t_{j} ; \Theta\right) \xi\left(t_{j} ; \Theta\right), \\
& \frac{\partial^{2} L^{*}}{\partial \alpha \partial \sigma}=-\sum_{j=1}^{n} p_{1} p_{2} \phi_{1}\left(t_{j} ; \Theta\right) \psi_{2}\left(t_{j} ; \Theta\right) \xi\left(t_{j} ; \Theta\right) \text {, } \\
& \frac{\partial^{2} L^{*}}{\partial \beta^{2}}=\sum_{j=1}^{n} p_{1} \eta_{1}\left(t_{j} ; \Theta\right) \\
& \cdot\left[V_{3}\left(t_{j} ; \Theta\right)+\left[1-p_{1} \eta_{1}\left(t_{j} ; \Theta\right)\right] \phi_{2}^{2}\left(t_{j} ; \Theta\right)\right],
\end{aligned}
$$


TABLE 5

\begin{tabular}{lccccc}
\hline Parameter & $p_{1}$ & $\alpha$ & $\beta$ & $\mu$ & $\sigma$ \\
\hline $90 \%$ CIs & $0.111,0.516$ & $1.351,3.489$ & $0.732,2.544$ & $0.582,1.291$ & $0.887,1.502$ \\
\hline
\end{tabular}

$$
\begin{aligned}
& \frac{\partial^{2} L^{*}}{\partial \beta \partial \mu}=-\sum_{j=1}^{n} p_{1} p_{2} \phi_{2}\left(t_{j} ; \Theta\right) \psi_{1}\left(t_{j} ; \Theta\right) \xi\left(t_{j} ; \Theta\right), \\
& \frac{\partial^{2} L^{*}}{\partial \beta \partial \sigma}=-\sum_{j=1}^{n} p_{1} p_{2} \phi_{2}\left(t_{j} ; \Theta\right) \psi_{2}\left(t_{j} ; \Theta\right) \xi\left(t_{j} ; \Theta\right), \\
& \frac{\partial^{2} L^{*}}{\partial \mu^{2}} \\
& \quad=\sum_{j=1}^{n} p_{2} \eta_{2}\left(t_{j} ; \Theta\right)\left[-\sigma^{-2}+\left[1-p_{2} \eta_{2}\left(t_{j} ; \Theta\right)\right] \psi_{1}^{2}\left(t_{j} ; \Theta\right)\right],
\end{aligned}
$$$$
\frac{\partial^{2} L^{*}}{\partial \mu \partial \sigma}
$$$$
=\sum_{j=1}^{n} p_{2} \eta_{2}\left(t_{j} ; \Theta\right)\left[\ell_{1}\left(t_{j} ; \Theta\right)+\left[1-p_{2} \eta_{2}\left(t_{j} ; \Theta\right)\right]\right.
$$$$
\left.\cdot \psi_{1}\left(t_{j} ; \Theta\right) \psi_{2}\left(t_{j} ; \Theta\right)\right]
$$$$
\frac{\partial^{2} L^{*}}{\partial \sigma^{2}}=\sum_{j=1}^{n} p_{2} \eta_{2}\left(t_{j} ; \Theta\right)
$$$$
\cdot\left[\ell_{2}\left(t_{j} ; \Theta\right)+\left[1-p_{2} \eta_{2}\left(t_{j} ; \Theta\right)\right] \psi_{2}^{2}\left(t_{j} ; \Theta\right)\right],
$$

where $\xi\left(t_{j} ; \Theta\right), V_{1}\left(t_{j} ; \Theta\right), V_{2}\left(t_{j} ; \Theta\right), V_{3}\left(t_{j} ; \Theta\right), \ell_{1}\left(t_{j} ; \Theta\right)$, and $\ell_{2}\left(t_{j} ; \Theta\right)$ are given, respectively, by

$$
\begin{aligned}
& \xi\left(t_{j} ; \Theta\right)=\frac{f_{1}\left(t_{j} ; \Theta_{1}\right) f_{2}\left(t_{j} ; \Theta_{2}\right)}{f^{2}\left(t_{j} ; \Theta\right)}, \\
& V_{1}\left(t_{j} ; \Theta\right)=\beta \alpha^{-2}-\beta(\beta+1) \alpha^{-(\beta+2)} t_{j}^{-\beta}, \\
& V_{2}\left(t_{j} ; \Theta\right)=-\alpha^{-1}+\alpha^{-(\beta+1)} t_{j}^{-\beta}-\beta \alpha^{-(\beta+1)} t_{j}^{-\beta} \log \left(\alpha t_{j}\right), \\
& V_{3}\left(t_{j} ; \Theta\right)=-\left(\beta^{-2}+\left(\alpha t_{j}\right)^{-\beta}\left(\log \left(\alpha t_{j}\right)\right)^{2}\right), \\
& \ell_{1}\left(t_{j} ; \Theta\right)=-\frac{2\left(\log t_{j}-\mu\right)}{\sigma^{3}}, \\
& \ell_{2}\left(t_{j} ; \Theta\right)=-3\left(\log t_{j}-\mu\right)^{2} \sigma^{-4}+\sigma^{-2},
\end{aligned}
$$

with $f\left(t_{j} ; \Theta\right), f_{1}\left(t_{j} ; \Theta_{1}\right), f_{2}\left(t_{j} ; \Theta_{2}\right), \omega\left(t_{j} ; \Theta\right), \phi_{1}\left(t_{j} ; \Theta\right), \phi_{2}\left(t_{j}\right.$; $\Theta), \eta_{1}\left(t_{j} ; \Theta\right), \eta_{2}\left(t_{j} ; \Theta\right), \psi_{1}\left(t_{j} ; \Theta\right)$, and $\psi_{2}\left(t_{j} ; \Theta\right)$ being as given in (1), (2), (3), and (23)-(29), respectively.

The Fisher information matrix $I(\Theta)$ can then be obtained as $\left(\left(-\partial^{2} L^{*}(\Theta) / \partial \theta_{i} \partial \theta_{j}\right)\right)$, and based on an observed data, an estimate of it can be obtained from the expressions in (A.2) evaluated at $\widehat{\Theta}$.

\section{Conflict of Interests}

The authors declare that there is no conflict of interests regarding the publication of this paper.

\section{Acknowledgments}

The authors would like to thank the referees for their helpful comments, which improved the presentation of the paper. The authors would like to extend their sincere appreciation to the Deanship of Scientific Research at King Saud University for its funding this research group no. (RG-1435-056).

\section{References}

[1] E. K. Al-Hussaini and K. S. Sultan, "Reliability and hazard based on finite mixture models," in Advances in Reliability, N. Balakrishnan and C. R. Rao, Eds., vol. 20 of Handbook of Statistics, pp. 139-183, Amsterdam, The Netherlands, North-Holland, 2001.

[2] B. S. Everitt and D. J. Hand, Finite Mixture Distributions, Chapman \& Hall, London, UK, 1981.

[3] D. M. Titterington, A. F. Smith, and U. E. Makov, Statistical Analysis of Finite Mixture Distributions, Wiley Series in Probability and Mathematical Statistics: Applied Probability and Statistics, John Wiley \& Sons, Chichester, UK, 1985.

[4] G. J. McLachlan and K. E. Basford, Mixture Models: Applications to Clustering, Marcel Dekker, New York, NY, USA, 1988.

[5] B. G. Lindsay, Mixture Models: Theory, Geometry and Applications, The Institute of Mathematical Statistics, Hayward, Calif, USA, 1995.

[6] G. J. McLachlan and T. Krishnan, The EM Algorithm and Extensions, John Wiley \& Sons, New York, NY, USA, 1997.

[7] G. McLachlan and D. Peel, Finite Mixture Models, John Wiley \& Sons, New York, NY, USA, 2000.

[8] N. L. Johnson, S. Kotz, and N. Balakrishnan, Continuous Univariate Distributions, Vol. 1, Wiley Series in Probability and Mathematical Statistics: Applied Probability and Statistics, John Wiley \& Sons, New York, NY, USA, 2nd edition, 1994.

[9] E. L. Crow and K. Shimizu, Eds., The Lognormal Distribution, Marcel Dekker, New York, NY, USA, 1988.

[10] J. S. Kim and B.-J. Yum, "Selection between Weibull and lognormal distributions: a comparative simulation study, Computational Statistics \& Data Analysis, vol. 53, no. 2, pp. 477-485, 2008.

[11] C.-T. Lin, S. J. Wu, and N. Balakrishnan, "Planning life tests with progressively Type-I interval censored data from the lognormal distribution," Journal of Statistical Planning and Inference, vol. 139, no. 1, pp. 54-61, 2009.

[12] E. K. Al-Hussaini, M. A. Mousa, and K. S. Sultan, "Parametric and nonparametric estimation of $P(Y<X)$ for finite mixtures of lognormal components," Communications in Statistics. Theory and Methods, vol. 26, no. 5, pp. 1269-1289, 1997.

[13] A. Drapella, "The complementary weibull distribution: unknown or just forgotten?” Quality and Reliability Engineering International, vol. 9, no. 4, pp. 383-385, 1993. 
[14] K. S. Sultan, M. A. Ismail, and A. S. Al-Moisheer, "Mixture of two inverse Weibull distributions: properties and estimation," Computational Statistics \& Data Analysis, vol. 51, no. 11, pp. 5377-5387, 2007.

[15] K. S. Sultan, M. A. Ismail, and A. S. Al-Moisheer, "Testing the number of components of the mixture of two inverse Weibull distributions," International Journal of Computer Mathematics, vol. 86, no. 4, pp. 693-702, 2009.

[16] A. Z. Keller, A. R. R. Kamath, and U. D. Perera, "Reliability analysis of CNC machine tools," Reliability Engineering, vol. 3, no. 6, pp. 449-473, 1982.

[17] R. Jiang, D. N. P. Murthy, and P. Ji, "Models involving two inverse Weibull distributions," Reliability Engineering \& System Safety, vol. 73, no. 1, pp. 73-81, 2001.

[18] W. H. Von Alven, Reliability Engineering by ARINC, Prentice Hall, Upper Saddle River, NJ, USA, 1964. 


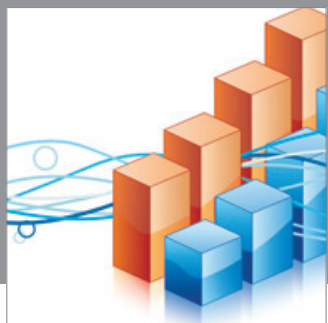

Advances in

Operations Research

mansans

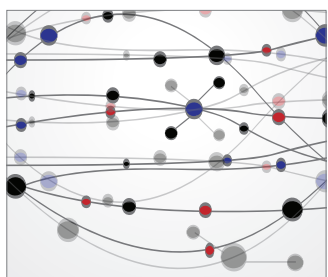

The Scientific World Journal
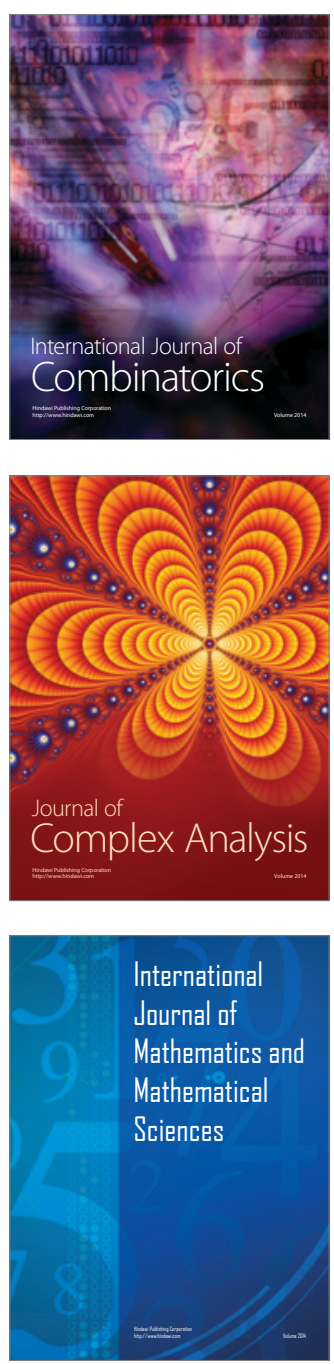
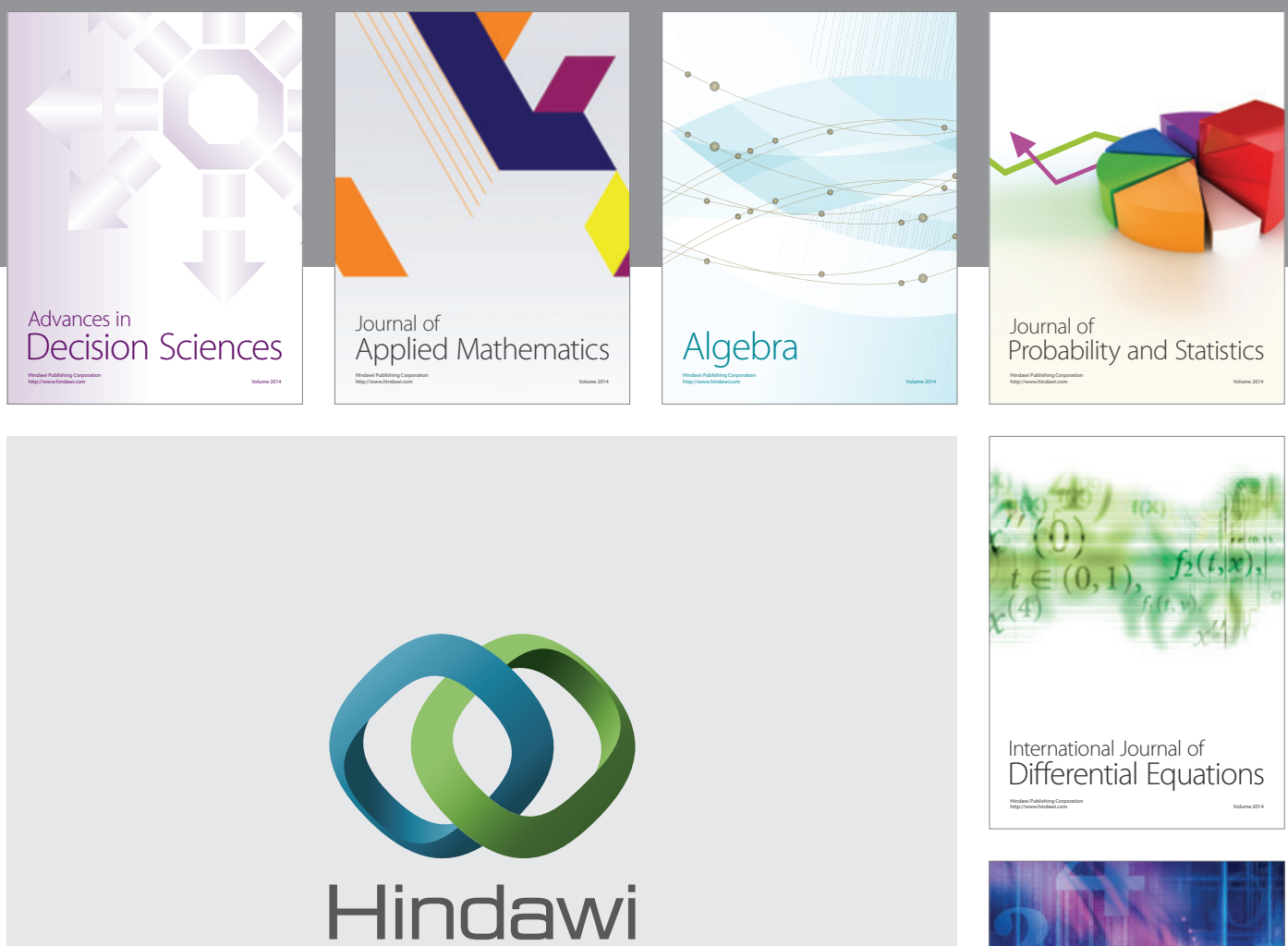

Submit your manuscripts at http://www.hindawi.com
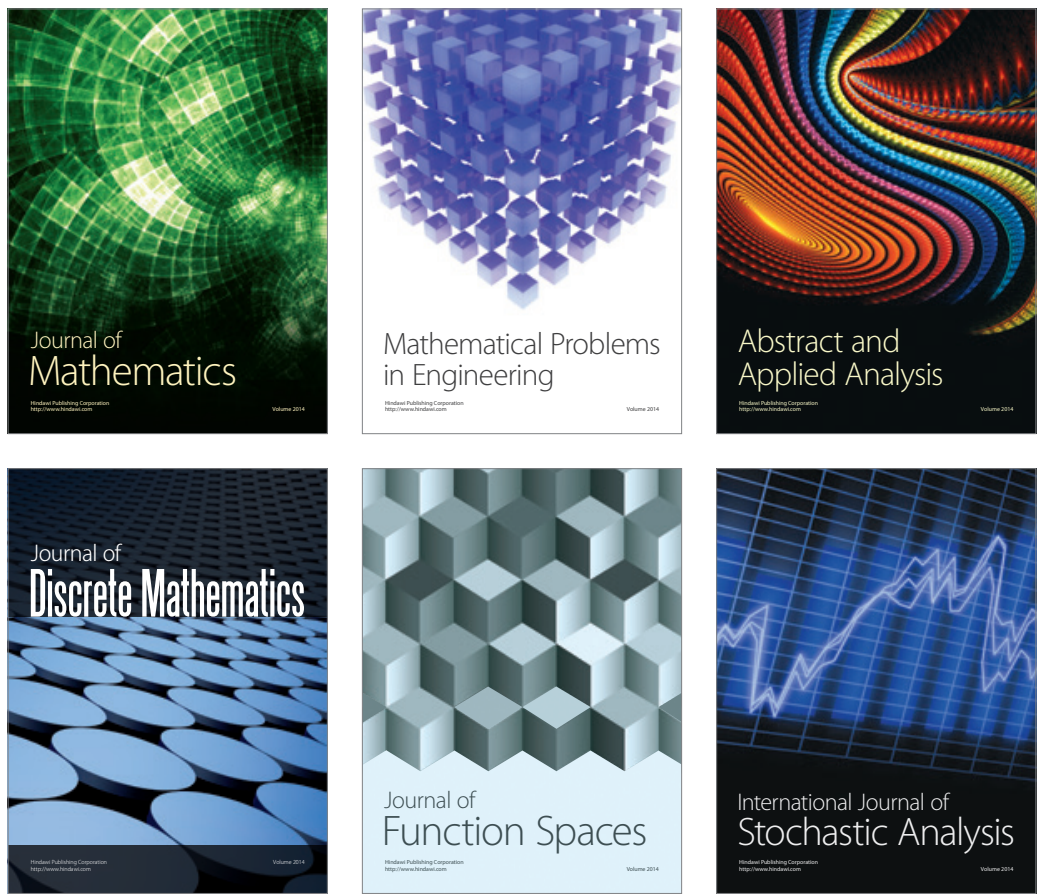

Journal of

Function Spaces

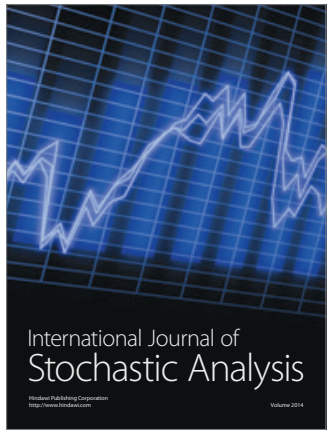

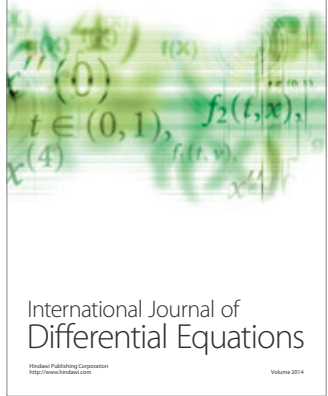
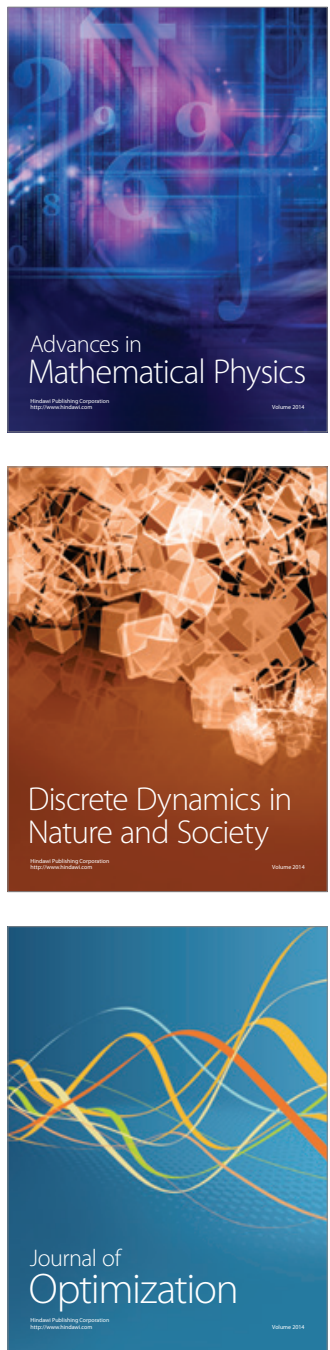